\title{
Fermat collocation method with convergence analysis of Volterra integro- differential equations
}

\author{
amany mohamed ${ }^{1}$ \\ ${ }^{1}$ Helwan University Faculty of Science
}

October 23, 2020

\begin{abstract}
this article presents the algorithm for solving a Volterra integro-differential equation using Fermat collocation method. The constructing and the properties of Fermat expansion were displayed. The method depends on modifying the Volterra integrodifferential equation with initial conditions to a system of equations in the coefficients, which must be determined, of the expansion. We use properties of the matrices to do this modification. We investigate accurately the convergence and error analysis of the problem. Some examples are solved using this algorithm and the absolute errors were compared with others. The results proved the method is the most accuracy of the others.
\end{abstract}

\section{Hosted file}

fermat.pdf available at https://authorea.com/users/369685/articles/488496-fermat-collocationmethod-with-convergence-analysis-of-volterra-integro-differential-equations 